\title{
ON LIE RINGS SATISFYING THE FOURTH ENGEL CONDITION
}

\author{
MOHAN S. PUTCHA
}

ABSTRACT. In this paper we prove that a Lie ring of characteristic prime to 2, 3 and 5, satisfying the fourth Engel condition, is nilpotent.

1. Let $L$ be a Lie ring satisfying the fourth Engel condition, that is, for any $a, x$ in $L, a x^{4}=0$. Higgins [3] proved that if $L$ has characteristic prime to $2,3,5$, and 7 , then $L$ is nilpotent. Bachmuth et al. [1] showed that if $L$ has characteristic 5 , then $L$ need not be nilpotent. The purpose of this note is to show that if $L$ has characteristic prime to 2, 3 and 5 , then $L$ is nilpotent.

2. Let $R$ be the (additive) endomorphism ring of $L$. Let $D$ be the subset consisting of the inner derivations of $L, X: a \rightarrow a x . D$ is a Lie ring under the product $(X, Y)=X Y-Y X . D$ is easily seen to be a homomorphic image of $L$ under the map $x \rightarrow X$.

THEOREM. A Lie ring $L$ of characteristic 7 , satisfying the fourth Engel condition is nilpotent.

Proof. For any $X, Y$ in $D$, the following relations are evident from Higgins [3, Theorem 4]:

$$
\begin{aligned}
X^{3} Y+2 X Y X^{2} & =0 . \\
X^{3} Y-4 X^{2} Y X+6 X Y X^{2}-4 Y X^{3} & =0 . \\
X^{3} Y^{3} & =-Y^{3} X^{3} . \\
Y X^{3} Y^{2} & =0 . \\
Y^{2} X^{3} Y & =-X^{3} Y^{3} .
\end{aligned}
$$

In the same paper Higgins discusses the process of linearization in his Lemma 1. Linearizing (1) we obtain,

$$
\sum_{i=1}^{18} Y_{1 \sigma_{i}} Y_{2 \sigma_{i}} Y_{3 \sigma_{i}} Y_{4 \sigma_{i}}=0 \text {. }
$$

The $\sigma_{i}$ 's are some fixed permutations of $\{1,2,3,4\}$, and $Y_{1}, Y_{2}, Y_{3}, Y_{4}$ are any elements of $D$.

Received by the editors September 1, 1970.

AMS 1969 subject classifications. Primary 1730.

Key words and phrases. Lie rings, Engel condition, exponent 7. 
We first show that $X^{3} Y^{3}=3(Y, X, X, X, Y, Y)$ and is hence in $D$.

$$
\begin{aligned}
(Y, X) & =Y X-X Y, \\
(Y, X, X) & =Y X^{2}-2 X Y X+X^{2} Y .
\end{aligned}
$$

Using (1) and (2) we obtain

$$
\begin{aligned}
&(Y, X, X, X)=Y X^{3}-3 X Y X^{2}+3 X^{2} Y X-X^{3} Y \\
&=Y X^{3}+\left(-4 X^{2} Y X+6 X Y X^{2}\right)-\left(2 X Y X^{2}+X^{3} Y\right) \\
&=Y X^{3}+\left(-4 X^{2} Y X+6 X Y X^{2}\right) \\
&=Y X^{3}+\left(4 Y X^{3}-X^{3} Y\right) \\
&=5 Y X^{3}-X^{3} Y, \\
&(Y, X, X, X, Y)=6 Y X^{3} Y-X^{3} Y^{2}-5 Y^{2} X^{3} .
\end{aligned}
$$

Using (3), (4) and (5) we obtain,

$(Y, X, X, X, Y, Y)$

$$
\begin{aligned}
& =6 Y X^{3} Y^{2}-X^{3} Y^{3}-5 Y^{2} X^{3} Y-6 Y^{2} X^{3} Y+Y X^{3} Y^{2}+5 Y^{3} X^{3} \\
& =5 X^{3} Y^{3} .
\end{aligned}
$$

Hence, $X^{3} Y^{3}=15 X^{3} Y^{3}=3(Y, X, X, X, Y, Y)$ is in $D$.

Given any eight elements $X_{1}, \cdots, X_{8}$ of $D$, let

$$
A_{1}=X_{1}^{3} X_{2}^{3}, \quad A_{2}=X_{3}^{3} X_{4}^{3}, \quad A_{3}=X_{5}^{3} X_{6}^{3}, \quad A_{4}=X_{7}^{3} X_{8}^{3}
$$

Then each $A$ is in $D$. Moreover, using (3) we have,

$$
A_{1} A_{2}=X_{1}^{3} X_{2}^{3} X_{3}^{3} X_{4}^{3}=X_{3}^{3} X_{1}^{3} X_{2}^{3} X_{4}^{3}=X_{3}^{3} X_{4}^{3} X_{1}^{3} X_{2}^{3}=A_{2} A_{1} \text {. }
$$

Substituting $A_{j}$ for $Y_{j}$ in (6) and using the commutativity of the $A_{j}$ 's we have

$$
18 A_{1} A_{2} A_{3} A_{4}=0 .
$$

Dividing by 18 we have

$$
A_{1} A_{2} A_{3} A_{4}=0
$$

Consequently,

$$
X_{1}^{3} X_{2}^{3} \cdots X_{8}^{3}=0 .
$$

It follows from Lemma 4 in Higgins [3] and his remark near the end, that $L$ is nilpotent.

In his thesis [4, p. 92] Walkup proved the following lemma, here stated in a special context: 
Lemma. Suppose:

(1) every Lie ring of characteristic prime to certain primes $p_{1}$, $p_{2}, \cdots, p_{s}$ satisfying the identical relations $f_{i}$ is nilpotent;

(2) every Lie ring of characteristic $p_{s}$ satisfying the same identical relations $f_{i}$ is nilpotent.

Then every Lie ring of characteristic prime to $p_{1}, p_{2}, \cdots, p_{s-1}$ satisfying the identical relations $f_{i}$ is nilpotent.

As a consequence we obtain the following corollary:

Corollary 1. A Lie ring of characteristic prime to 2, 3 and 5 satisfying the fourth Engel condition is nilpotent.

Let $G$ be a group of exponent 7 satisfying the fourth Engel congruence, $(g, h, h, h, h) \equiv 1 \bmod G_{6}$. The associated Lie ring of $G$ (see Hall [2] for definition) is of characteristic 7 and satisfies the fourth Engel condition and is therefore nilpotent. We have shown the following:

COROLlaRY 2. The associated Lie ring of a group of exponent 7, satisfying the fourth Engel congruence, is nilpotent.

The author is indebted to Dr. H. Y. Mochizuki and Dr. S. Bachmuth for many stimulating discussions.

\section{REFERENCES}

1. S. Bachmuth, H. Y. Mochizuki and D. W. Walkup, A nonsolvable group of exponent 5, Bull. Amer. Math. Soc. 76 (1970), 638-640.

2. M. Hall, The theory of groups, Macmillan, New York, 1959. MR 21 \#1996.

3. P. J. Higgins, Lie rings satisfying the Engel condition, Proc. Cambridge Philos. Soc. 50 (1954), 8-15. MR 15, 596.

4. D. W. Walkup, Lie rings satisfying Engel conditions, Thesis, University of Wisconsin, Madison, Wis., 1963, 154 pp., University Microfilms, Ann Arbor, Michigan.

University of California at Santa Barbara, Santa Barbara, California 93106 